\title{
1. Factor structure of the German version of the Pain Attitudes and Beliefs Scale for Physiotherapists (Study 1)
}

Emanuel Brunner ${ }^{1,2}$, Wim Dankaerts ${ }^{3}$, André Meichtry ${ }^{4}$, Kieran O’Sullivan ${ }^{5,6}$, SeSergio Baldew ${ }^{7}$, and Michel Probst ${ }^{1}$

\author{
${ }^{1}$ Adapted Physical Activity and Psychomotor Rehabilitation Research Unit, Department of \\ Rehabilitation Sciences, University of Leuven, Leuven, Belgium \\ ${ }^{2}$ Institute of Physiotherapy, Kantonsspital Winterthur, 8401 Winterthur, Switzerland \\ ${ }^{3}$ Musculoskeletal Rehabilitation Research Unit, Department of Rehabilitation Sciences, University of \\ Leuven, Leuven, Belgium \\ ${ }^{4}$ Institute of Physiotherapy, School of Health Professions, Zurich University of Applied Sciences, 8401 \\ Winterthur, Switzerland \\ ${ }^{5}$ Sports Spine Centre, Aspetar Orthopaedic and Sports Medicine Hospital, Qatar, Doha \\ ${ }^{6}$ Department of Clinical Therapies, University of Limerick, Limerick, Ireland \\ ${ }^{6}$ Faculty of Medical Sciences, Anton de Kom University of Suriname, Paramaribo, Suriname
}

Key words: physiotherapy, low back pain, attitudes, factor analysis, questionnaire

This study is accepted at Physiotherapy Theory and Practice:

Brunner E, Dankaerts W, Meichtry A, O'Sullivan K, Baldeq SS, Probst M. Factor structure of the German version of the Pain Attitudes and Beliefs Scale for Physiotherapists. 


\subsection{Abstract}

The Pain Attitudes and Beliefs Scale for Physiotherapists (PABS-PT) is a questionnaire that is frequently used to measure the treatment orientation of physiotherapists and other healthcare providers toward low back pain (LBP). Previous validation studies of the PABS-PT have reported consistently that exploratory factor analysis (EFA) yielded a 2-factor model with the factors "biomedical" and "biopsychosocial." However, there remain concerns regarding the composition of these two factors and the internal consistency of the "biopsychosocial" factor. The objective of this study was to replicate the previous validation study on the German PABS-PT. EFA was implemented to reexamine the underlying structure of the scale, and confirmatory factor analysis (CFA) was used to test the fit of the hypothesized 2-factor model. Results of the initial validation study were not replicated. EFA indicated that a 2-factor solution is an inadequate representation of the PABS-PT data, and CFA showed insufficient fit of the hypothesized 2-factor model to the PABS-PT data. Our results indicate a need for caution when using the PABS-PT to measure physiotherapists' orientation toward LBP, particularly in measuring the magnitude of a biopsychosocial orientation. 


\subsection{Introduction}

Research on the attitudes and beliefs of physiotherapists regarding the management of low back pain (LBP) has received much attention during recent years. Numerous studies have investigated the influence of attitudes and beliefs on the clinical management provided by physiotherapists and other healthcare providers (Dalkilinc et al., 2015; Eland et al., 2017; Houben et al., 2005; Laekeman et al., 2008; Mutsaers et al., 2014; Ostelo et al., 2003). Other studies have focused on the effect of educational programs on healthcare providers' orientation toward LBP (Domenech, Sanchez-Zuriaga, Segura-Orti, Espejo-Tort, \& Lison, 2011; Jacobs, Guildford, Travers, Davies, \& McCracken, 2016; Overmeer, Boersma, Denison, \& Linton, 2011). The Pain Attitudes and Beliefs Scale for Physiotherapists (PABS-PT) was developed to measure attitudes and beliefs or treatment orientations. The multidimensional structure of the PABS-PT may explain the popularity of this instrument for measuring two different sets of attitudes and beliefs: biomedical and biopsychosocial orientations. The biomedical approach postulates that all signs and symptoms are caused by tissue damage or physical pathology, whereas the biopsychosocial approach highlights the role of psychological and social factors in the development and maintenance of chronic musculoskeletal problems (Ostelo et al., 2003). A therapist with a strong biomedical orientation is less likely to implement current guidelines for the management of LBP and may even induce patients' fear or their beliefs that painful activities result in tissue damage (Ostelo \& Vlaeyen, 2008). Therefore, measuring healthcare providers' attitudes and beliefs regarding the management of LBP might be relevant.

The factor structure of the PABS-PT has been tested in several validation studies. In the initial developmental study by Ostelo et al. (2003)(Ostelo et al., 2003), the construct of the attitudes and beliefs of physiotherapists toward LBP management was measured by using a 31-item pool (Ostelo et al., 2003). After testing the performance of the item pool, several problematic items were discarded, and the subsequent exploratory factor analysis (EFA) yielded a 2 -factor model consisting of 20 items. Houben et al. (2005) amended this first version of the PABS-PT and reexamined the factor structure of the measurement scale(Houben et al., 2005). Instead of using the initially proposed 20 -item scale, the authors added five new items to the 31-item pool and replicated the factor analytical procedure described in 
the initial developmental study (Houben et al., 2005). Similar to the initial study, several measured items were eventually excluded, and EFA yielded a new 2-factor model that included 19 measured variables. Later validation studies used different item pools and excluded different items from the factor models fitted to the measurement scale (Dalkilinc et al., 2015; Eland et al., 2017; Laekeman et al., 2008; Mutsaers et al., 2014). In the validation study of the German language version of the PABS-PT, Laekeman et al. (2008) used the 36-item PABS-PT scale from Houben et al (2005) but included only a 17-item pool in the EFA(Laekeman et al., 2008). However, the two identified common factors significantly influenced only 14 PABS-PT items. Table 1 shows an overview of the different common factor models obtained in previous validation studies of the PABS-PT.

There remain concerns regarding the factor structure of the PABS-PT. The validity of the hypothesized 2-factor model might be problematic, because the factor models describe the underlying structure of different item pools, but not the structure of the PABS-PT that was used to measure physiotherapists' orientation toward LBP. Furthermore, the compositions of the biomedical and the biopsychosocial factors were different across previous validation studies of the PABS-PT, particularly regarding the biopsychosocial factor (see Table 1). Furthermore, the internal consistency of this "biopsychosocial" factor was found to be poor (Cronbach's alpha $=<0.60)$ in the models estimated using the German, Turkish, and Norwegian measurement scales (Dalkilinc et al., 2015; Eland et al., 2017; Laekeman et al., 2008). The actual fit of the hypothesized 2 -factor model to the PABS-PT remains unknown. Confirmatory factor analysis (CFA) allows the testing of specific hypotheses about the data(Finch \& West, 1997). However, this statistical method has never been used to test the fit of the hypothesized 2-factor model of the PABS-PT data. 
Table 1: Overview of the previous factor analysis studies of the PABS-PT

\begin{tabular}{|c|c|c|c|c|c|c|}
\hline & Ostelo et al, 2003 & $\begin{array}{l}\text { Houben et al, } \\
2005\end{array}$ & $\begin{array}{l}\text { Laekeman et } \\
\text { al, } 2008\end{array}$ & $\begin{array}{l}\text { Dalkilinc et al, } \\
2015\end{array}$ & $\begin{array}{l}\text { Mutsaers et al, } \\
2014\end{array}$ & $\begin{array}{l}\text { Eland et al, } \\
2016\end{array}$ \\
\hline Language: & Dutch & Dutch & German & Turkish & Dutch & Norwegian \\
\hline $\begin{array}{l}\text { Composition of the } \\
\text { scale: }\end{array}$ & 31-items & 36-items & 36-items & 31-items & 36-items & 36-items \\
\hline $\begin{array}{l}\text { Number of items } \\
\text { included for factor } \\
\text { analysis: }\end{array}$ & 20 & 19 & 17 & 13 & 15 & 19 \\
\hline $\begin{array}{l}\text { Factor composition a of } \\
\text { the factor } 1 \\
\text { "biomedical": }\end{array}$ & $\begin{array}{l}25,24,31,26,22 \\
30,10,13,14,9 \\
20,5,23,4\end{array}$ & $\begin{array}{l}31,25,10,22 \\
30,14,24,23 \\
20,35\end{array}$ & $\begin{array}{l}\mathbf{2 5}, \mathbf{3 1}, \mathbf{2 3}, 10 \\
30,26,35,4 \\
5,14\end{array}$ & $\begin{array}{l}24,23,31,25,28 \\
14,9\end{array}$ & $\begin{array}{l}25,23,24,29 \\
31,10,20\end{array}$ & $\begin{array}{l}25,20,30,31 \\
24,10,23,14, \\
26,4,5,35,22\end{array}$ \\
\hline $\begin{array}{l}\text { Internal consistency } \\
\text { of the factor } 1 \text { : }\end{array}$ & $\alpha=0.83$ & $\alpha=0.73$ & $\alpha=0.77$ & $\alpha=0.72$ & $\alpha=0.75$ & $\alpha=0.79$ \\
\hline $\begin{array}{l}\text { Factor compositiona of } \\
\text { the factor } 2 \\
\text { "biopsychosocial": }\end{array}$ & $7,12,6,3,27,11$ & $\begin{array}{l}33,11,29,34 \\
17,7,12,27,6\end{array}$ & $34,29,19,11$ & $\begin{array}{l}20,15,13,12,17 \\
30\end{array}$ & $\begin{array}{l}12,36,17,6,7 \\
33,27,3\end{array}$ & $\begin{array}{l}11,33,29,34 \\
17,22\end{array}$ \\
\hline $\begin{array}{l}\text { Internal consistency } \\
\text { of the factor } 2 \text { : }\end{array}$ & $\alpha=0.54$ & $\alpha=0.68$ & $\alpha=0.58$ & $\alpha=0.59$ & $\alpha=0.73$ & $\alpha=0.55$ \\
\hline
\end{tabular}

a the factor composition relates to the PABS-PT items influenced by the factor; ${ }^{\circ}$ Cronbach's alpha; numbers in bold indicate items which were consistent across previous factor models; for clarity of presentation, items are sorted in descending order based on the magnitude of loadings on measured items 
Previous studies have mentioned that the PABS-PT is still in a developmental phase (Bishop, Thomas, \& Foster, 2007; Mutsaers, Peters, Pool-Goudzwaard, Koes, \& Verhagen, 2012). Nevertheless, the PABS-PT has never been revised, and confusion remains regarding the factor structure and composition of this frequently used questionnaire. We aimed to replicate the previous validation study on the German version of the PABS-PT (Laekeman et al., 2008), and implement EFA and CFA to examine the factor structure of the questionnaire. The first objective was to examine the factor structure of the 36 -item scale, which was used to measure the attitudes and beliefs of physiotherapists. The second objective was to reexamine the underlying structure of the 17-item pool, which was included in the previous validation study of the German language version of the PABS-PT (Laekeman et al., 2008). The third objective was to test the fit of the hypothesized 2-factor model. For the latter objective, CFA was implemented to test the fit of the hypothesized model including the 14 items that were influenced by factor model, as identified from the EFA in the initial factor analysis of the German PABS-PT (Laekeman et al., 2008).

\subsection{Methods}

\section{Procedure and Participants}

General physiotherapists $(\mathrm{N}=1066)$ and physiotherapy students $(\mathrm{N}=111)$ working or studying in the German-speaking part of Switzerland were recruited for this crosssectional survey study. The aim was to recruit participants similar to the sample included in the previous validation study (Laekeman, Sitter, and Basler, 2008), which was done in Germany. The email addresses of the general physiotherapists were derived from an online database provided by the Swiss Physiotherapy Association. To be eligible for inclusion in the survey, the physiotherapists had to be treating LBP patients in their clinical practice (>1 patient monthly). The physiotherapy students were third year undergraduates who had received training in LBP management. Online questionnaires were sent by e-mail, with one reminder e-mail sent two weeks later. The study was approved by the ethics committee of the Canton of Zurich. 


\section{Measures}

Demographics: The sociodemographic and professional characteristics of the participants were recorded, such as age, sex, years of experience in treating LBP patients, specialization in LBP management, and the frequency with which they treated LBP patients in their individual clinical practice.

PABS-PT: The participants completed the German 36-item PABS-PT scale, which was translated from Dutch by using the forward-backward procedure (Laekeman et al., 2008). The measurement scale had a Likert-type response format with six answer categories: 1 = totally disagree; 2 = largely disagree; 3 = disagree to some extent; 4 $=$ agree to some extent; $5=$ largely agree; and $6=$ totally agree. An example of a "biomedical" statement is item 25: "Increased pain indicates new tissue damage or the spread of existing damage". An example of a "biopsychosocial" statement is item 34: "Exercises that may be back straining should not be avoided during the treatment". Higher scores represent stronger agreement with the statement.

\section{Statistical Analysis}

Statistical analysis was carried out with the psych package R, version 2.14.1 (R Core Team, 2014). Data means, standard deviations, skewness, and kurtosis were calculated to evaluate the performance of each item.

Exploratory Factor Analysis: EFA was applied on two different item pools; first, on the 36 measured items; and second, on the 17-item pool. The adequacy of the empirical correlation matrix for EFA was verified by using the Kaiser-Meyer-Olkin (KMO) factor. The aim of EFA is to account for, or "explain," the covariance matrix by a minimum, or at least a small number, of unobserved or latent common factors (Lawley, 1963). The data $\mathrm{X}$ consists of $n$ observations of a $p$-dimensional centred vector $(p=36)$ of indicators (variables). A $k$-factor model $(k<p)$ for the $i$-th $p$-dimensional observation vector $X_{i}$ is given by:

$$
\begin{gathered}
X_{i 1}=\lambda_{11} f_{i 1}+\ldots+\lambda_{1 /} f_{i l}+\ldots+\lambda_{1 k} f_{i k}+u_{i 1} \\
\ldots=\ldots \\
X_{i j}=\lambda_{j 1} f_{i 1}+\ldots+\lambda_{j l} f_{i l}+\ldots+\lambda_{j k} f_{i k}+u_{i j} \\
\ldots=\ldots \\
X_{i p}=\lambda_{p 1} f_{i 1}+\ldots+\lambda_{p l} f_{i l}+\ldots+\lambda_{p k} f_{i k}+u_{i p},
\end{gathered}
$$


with $i=1, \ldots, \mathrm{n} ; j=1, \ldots, p$; and $l=1, \ldots, \mathrm{k}$. The factor scores $f_{i}=\left(f_{i 1}, \ldots, f_{i k}\right)^{T}$ are the unobserved scores on the common factors; $u_{i}=\left(u_{i 1}, \ldots, u_{i p}\right)^{T}$ denotes the specific factors or uniquenesses representing the source of variation (unique factor), with the $j$-th component of $u_{i}$ affecting only the corresponding indicator $X_{i j}$; and $\lambda_{j l}$ refers to the factor loadings of the $j$-th indicator on the $I$-th factor.

Correlation matrix: Pairwise deletion of missing items was used to compute the empirical correlation matrix. In EFA, the eigenvalues of the estimated reduced correlation matrix are computed. Squared multiple correlations (SMC) were used as initial communality estimates.

Determining the number of factors to retain: Parallel analysis, the Kaiser criterion (eigenvalue > mean eigenvalues), and Cattell's scree test were used in combination with non-graphic tests (optimal coordinates and acceleration factor) to determine the number of factors to retain in the common factor model. Additionally, the likelihood ratio statistic was used to test the model fit.

Parallel analysis is based on a comparison between eigenvalues obtained from sample data and eigenvalues that one would expect to obtain from completely random data (Horn, 1965). A model is specified with the same number of common factors as real eigenvalues (sample data) that are greater than the eigenvalues from random data. For the Kaiser criterion, eigenvalues from the reduced correlation matrix are computed. Eigenvalues derived from the reduced correlation matrix sum up to less than $p$, and therefore, the criterion eigenvalues > mean eigenvalues must be used in EFA. In the scree test, eigenvalues from the reduced correlation matrix are computed and plotted in descending order. The graph of eigenvalues is then examined to identify the last substantial drop in the line.

Non-graphic or numerical solutions to the subjective scree test have been proposed: the acceleration factor and the optimal coordinates index (Raîche, Walls, Magis, Riopel, \& Blais, 2013). The acceleration factor indicates the location of the elbow of the scree plot. It corresponds to the acceleration of the curve (e.g., the second derivative). The optimal coordinates are the extrapolated coordinates of the previous eigenvalues that allow the observed eigenvalue to go beyond this extrapolation. The extrapolation is made through linear regression by using the last eigenvalue coordinates and the $k+1$ eigenvalue coordinates. 
In the likelihood ratio framework, the likelihood ratio test can be used to compare the fit of two nested models under the assumption of a normal distribution of the data. Thereby, we tested if the model with $k=1,2, \ldots$ factors was sufficient by constructing a likelihood ratio test that compared the null hypothesis $\left(\mathrm{H}_{0}=\right.$ " $k$ factors are sufficient") with the alternative hypothesis ( $\mathrm{H}_{1}=$ " $k$ factors are not sufficient"). The test was applied to a series of numbers of factors $(k)$, starting with one and continuing until a nonsignificant test statistic was obtained, indicating that the common factor model with the corresponding number of factors was not rejected (Leandre R. Fabrigar, Wegener, MacCallum, \& Strahan, 1999). The likelihood ratio statistic was approximately chi-square distributed, with degrees of freedom equal to the number of unique elements in the covariance matrix $(\Sigma)$ minus the number of variables estimated. The significance level was set at alpha $=0.05$.

Factor rotation: Principal axis was used as the method of factor extraction. The extracted factors are likely to be related to one another if the model is used to estimate different sets of attitudes and beliefs. Therefore, oblique factor rotation by using the oblimin criterion was applied because this procedure allowed correlations among factors.

\section{Confirmatory Factor Analysis}

CFA was carried out to examine the validity of the hypothesized 2-factor model, including the 14 items obtained in the initial factor analysis on the German version of the PABS-PT (Laekeman et al., 2008). The hypothesized model included two latent or common factors and 14 measured items, with 10 items loading on factor 1 labeled as "biomedical" (items $25,31,23,10,30,26,35,4,5$, and 14), and 4 items loading on factor 2 labeled as "biopsychosocial" (items 34, 29, 19, and 11). The model fit was assessed by applying the chi-square test statistic and the comparative fit index (CFI). The chi-square test statistic, which was the primary test for the model, describes the difference between the measured covariance matrix and the model covariance matrix. The fit of the model was considered good if the chi-square test statistic was not significant ( $>0.05$ ) (MacCallum, Browne, \& Sugawara, 1996). The CFI describes the extent to which the data are better fitted to the hypothesized model than to the baseline model (null model). CFI values close to 1.0 indicate a virtually perfect fit of the data to the hypothesized model. The criteria for a good model fit were defined 
according to $\mathrm{Hu}$ and Bentler (1999)(Hu \& Bentler, 1999), with CFI values of 0.95 or greater indicating a good model fit.

\subsection{Results}

Questionnaires from 358 general physiotherapists (33.6\%) and 80 physiotherapy students $(72.1 \%)$ were returned. Forty questionnaires were excluded from later analysis due to missing responses for $>10 \%$ of items on the PABS-PT $(\mathrm{N}=36)$ or due to physiotherapists self-reporting not regularly treating patients with $\operatorname{LBP}(\mathrm{N}=4)$. The final analysis thus included 398 questionnaires: 318 (79.9\%) from general physiotherapists and $80(20.1 \%)$ from physiotherapy students. Questionnaires from 371 (93.2\%) participants had no missing values on the PABS-PT.

The majority of the 318 general physiotherapists were female ( $N=219,68.4 \%)$, worked in private physiotherapy practices $(\mathrm{N}=280,88.1 \%)$, and specialized in manual therapy ( $\mathrm{N}=232,72.5 \%$ ). The mean (SD) age of the general physiotherapists was 44.7 (10.3) years, and their mean (SD) length of experience in treating patients with LBP was 18.9 (10.3) years. The physiotherapy students had a mean (SD) age of 24.0 (2.7) years and were predominantly female (90\%). Table 2 shows the characteristics of the study participants. Table 3 presents the descriptive statistics for all the PABS-PT items. 
Table 2: Characteristics of participants included for the analysis $(\mathrm{N}=398)$

\begin{tabular}{|c|c|c|}
\hline & General PT & Student PT \\
\hline $\mathrm{N}:$ & 318 & 80 \\
\hline Gender: female (\%) & $219(68.4 \%)$ & $72(90.0 \%)$ \\
\hline Age: mean (SD) & $44.7(10.3)$ & $24.0(2.7)$ \\
\hline Years of experience in LBP management: mean (SD) & $18.9(10.3)$ & na \\
\hline Frequency of patients with LBP in clinic: N (\%) & & na \\
\hline One per day & $257(80.3)$ & \\
\hline One per week & $57(17.8)$ & \\
\hline One per month & $4(1.3)$ & \\
\hline Work setting: N (\%) & & na \\
\hline Private practice & $280(88.1)$ & \\
\hline Hospital & $29(9.1)$ & \\
\hline Rehabilitation centre & $6(1.9)$ & \\
\hline Others & $3(0.9)$ & \\
\hline Specialisation in LBP management: $\mathrm{N}(\%)^{1}$ & & na \\
\hline Manual Therapy & $232(72.5)$ & \\
\hline McKenzie & $47(14.7)$ & \\
\hline Others & $54(16.9)$ & \\
\hline No specialisation & 57 (17.8) & \\
\hline
\end{tabular}

\section{EFA on the 36-item PABS-PT scale}

The $\mathrm{KMO}$ test value was 0.83 , indicating that the correlation matrix was adequate for factor analysis. The mean eigenvalue of the 36 vectors (items) derived from the reduced correlation matrix was 0.239 (range, -0.357 to 5.332 ). The Kaiser criterion (eigenvalue $>$ mean eigenvalues) suggested the extraction of 10 factors $(k=10)$. The eigenvalues of these factors were: $5.332,1.532,1.055,0.897,0.756,0.618,0.506$, $0.440,0.319$, and 0.284 , with the next lower eigenvalue being 0.238 . Parallel analysis and optimal coordinates both suggested the extraction of eight factors ( $k=$ 8). In the likelihood ratio test, only when $k=8$ was the null hypothesis " $k$ factors are sufficient" not rejected $(p=0.05)$. Figure 1 (left) presents a graphic summary, 
including the eigenvalues computed from the reduced correlation matrix. The scree plot included one very large eigenvalue and a second potential common factor. However, the acceleration factor showed the elbow of the curve on the second eigenvalue, indicating the extraction of only one factor. Parallel analysis, optimal coordinates, and the likelihood ratio test identified eight common factors. For the suggested 8-factor model, the pattern matrix after oblique rotation showed a large factor 1 with significant loadings $(>0.30)$ to 8 measured PABS-PT items $(25,10,31$, $15,20,24,26$, and 30 ). The descending factors included several negative loadings to measured items and several PABS-PT items were not influenced by the identified common factors.

Table 3: Descriptive statistics of the 36-item pool $(N=398)$

\begin{tabular}{|c|c|c|c|c|c|}
\hline $\mathrm{Nr}$. & Statement & M & SD & skew & kurt \\
\hline 1 & $\begin{array}{l}\text { Back pain sufferers should refrain from all physical } \\
\text { activity in order to avoid injury }\end{array}$ & 1.6 & 0.90 & 1.64 & 2.49 \\
\hline 2 & Good posture prevents back pain & 4.5 & 1.11 & -1.05 & 1.44 \\
\hline 3 & $\begin{array}{l}\text { Knowledge of the tissue damage is not necessary for } \\
\text { effective therapy }\end{array}$ & 2.4 & 1.34 & 0.77 & -0.34 \\
\hline 4 & $\begin{array}{l}\text { Reduction of daily physical exertion is a significant factor } \\
\text { in treating back pain }\end{array}$ & 2.6 & 1.18 & 0.34 & -0.81 \\
\hline 5 & $\begin{array}{l}\text { Not enough effort is made to find the underlying organic } \\
\text { causes of back pain }\end{array}$ & 3.3 & 1.31 & 0.17 & -0.77 \\
\hline 6 & $\begin{array}{l}\text { Mental stress can cause back pain even in the absence } \\
\text { of tissue damage }\end{array}$ & 4.6 & 1.14 & -0.87 & 0.83 \\
\hline 7 & The cause of back pain is unknown & 2.8 & 1.21 & 0.20 & -0.88 \\
\hline 8 & Unilateral physical stress is not a cause of back pain & 2.4 & 1.22 & 0.84 & 0.10 \\
\hline 9 & $\begin{array}{l}\text { Patients who have suffered back pain should avoid } \\
\text { activities that stress the back }\end{array}$ & 2.5 & 1.13 & 0.46 & -0.64 \\
\hline 10 & Pain is a nociceptive stimulus, indicating tissue damage & 3.2 & 1.23 & -0.28 & -0.77 \\
\hline 11 & $\begin{array}{l}\text { A patient suffering from severe back pain will benefit } \\
\text { from physical exercise }\end{array}$ & 4.0 & 1.20 & -0.48 & -0.20 \\
\hline 12 & $\begin{array}{l}\text { Functional limitations associated with back pain are the } \\
\text { result of psychosocial factors }\end{array}$ & 3.2 & 1.21 & -0.25 & -0.75 \\
\hline 13 & $\begin{array}{l}\text { The best advice for back pain is: "Take care" and "Make } \\
\text { no unnecessary movements" }\end{array}$ & 1.9 & 0.92 & 0.99 & 0.89 \\
\hline 14 & $\begin{array}{l}\text { Patients with back pain should preferably practice only } \\
\text { pain free movements }\end{array}$ & 3.6 & 1.19 & -0.25 & -0.76 \\
\hline
\end{tabular}


15 Back pain indicates that there is something dangerously wrong with the back

16 The way patients view their pain influences the progress of the symptoms

17 Therapy may have been successful even if pain remains

18 Therapy can completely alleviate the functional symptoms caused by back pain

19 If ADL activities cause more back pain, this is not dangerous

20 Back pain indicates the presence of organic injury

21 Sport should not be recommended for patients with back pain

22 If back pain increases in severity, I immediately adjust the intensity of my treatment accordingly

23 If therapy does not result in a reduction in back pain, there is a high risk of severe restrictions in the long term

24 Pain reduction is a precondition for the restoration of normal functioning
25 Increased pain indicates new tissue damage or the spread of existing damage

26 It is the task of the physiotherapist to remove the cause of back pain

27 There is no effective treatment to eliminate back pain

$2.1 \quad 1.14 \quad 1.09 \quad 0.72$

28 TENS and/or back braces support functional recovery

$3.2 \quad 1.33 \quad-0.11 \quad-0.99$

29 Even if the pain has worsened, the intensity of the next treatment can be increased

30 If patients complain of pain during exercise, I worry that damage is being caused

31 The severity of tissue damage determines the level of pain

$\begin{array}{llll}3.4 & 1.33 & -0.18 & -0.87 \\ 4.0 & 1.36 & -0.57 & -0.50 \\ 2.9 & 1.16 & 0.17 & -0.74 \\ 2.9 & 1.30 & 0.23 & -0.83\end{array}$

$\begin{array}{llll}4.4 & 1.07 & -0.71 \quad 0.51\end{array}$

$2.6 \quad 1.11 \quad 0.52 \quad-0.21$

$2.5 \quad 1.34 \quad 0.57 \quad-0.84$

32 A rapid resumption of daily activities is an important goal $\quad \begin{array}{llll}5.3 & 0.88 & -1.78 & 4.36\end{array}$ of the treatment

33 Learning to cope with stress promotes recovery from

$\begin{array}{llll}5.0 & 0.86 & -0.62 & 0.74\end{array}$
back pain

34 Exercises that may be back straining should not be avoided during the treatment

35 In the long run, patients with back pain have a higher risk of developing spinal impairments

36 In back pain, imaging tests are unnecessary

$\begin{array}{llll}4.7 & 1.04 & -0.74 & 0.56\end{array}$

$\begin{array}{llll}3.4 & 1.41 & -0.14 & -0.86\end{array}$

$2.8 \quad 1.31 \quad 0.20 \quad-1.00$


$\mathrm{N}$ = sample size; $\mathrm{M}$ = mean; $\mathrm{SD}$ = standard deviation; skew = skewness; kurt = kurtosis. Higher scores on the PABS-PT items represent strong agreement with the statement. The Likert-type responses ranged from $1=$ totally disagree, to $6=$ totally agree.

\section{EFA on the 17-item pool}

The KMO test value of the 17 items was 0.83 . The mean eigenvalue of the 17 vectors (items) derived from the reduced correlation matrix was 0.226 (range, -0.290 to 3.258). The Kaiser criterion (eigenvalue $>$ mean eigenvalues) suggested the extraction of four factors $(k=4)$. The eigenvalues of these factors were: $3.258,0.648$, 0.508 , and 0.2918 , with the next lower eigenvalue being 0.220 . Figure 1 (right) shows the scree plot of the eigenvalues computed from the reduced correlation matrix. This pattern of eigenvalues is similar to the previous scree plot from 36 items indicating one very large eigenvalue. Similar to the previous scree plot, the acceleration factor identified the elbow of the curve on the second eigenvalue, suggesting that one factor be retained. Parallel analysis and optimal coordinates both suggested specifying a 5 -factor model. Furthermore, in the likelihood ratio test, only when $k=5$ was the null hypothesis " $k$ factors are sufficient" not rejected $(p=0.16)$. For the specified 5 -factor model, the pattern matrix after oblique rotation showed a factor 1 with four significant loadings $(>0.30)$ to the measured items $(31,25,10$, and 26$)$.

\section{CFA testing the fit of the hypothesized 2-factor model}

For the predefined 2-factor model consisting of the selected 14 items, which matched the two common factors identified in the previous validation study (Laekeman et al., 2008 ), the chi-square test statistic was significant ( $p$-value $=0.000$ ), indicating a poor fit of the hypothesized 2-factor model from the initial validation study (MacCallum et al., 1996). Additionally, the CFI value was 0.888 , which was below the predefined cutoff (>0.95) for a good model fit (Hu \& Bentler, 1999). 
Figure 1: Solutions for the factor retention decision from EFA on the PABS-PT. The graph on the left showing solutions from EFA on the 36-item PABS-PT, and the graph on the right showing solutions from EFA on the 17 -item pool.
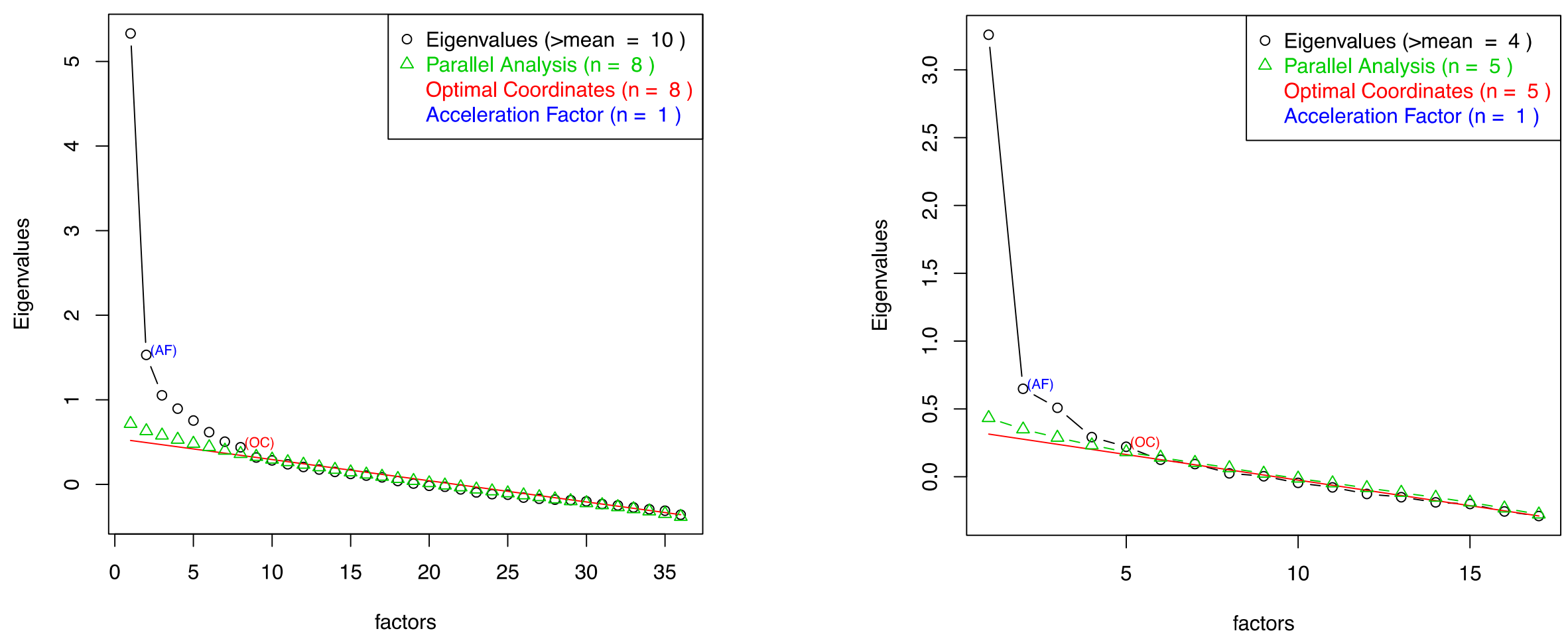

$\mathrm{OC}=$ optimal coordinates; $\mathrm{AF}$ = acceleration factor; eigenvalues estimated from the reduced correlation matrix.

The graphs show computed eigenvalues from the reduced correlation matrix plotted in descending order. Results from the four methods used for the factor retention decision illustrate that no method suggested to specify a 2-factor model on the different pools of PABS-PT items. 


\subsection{Discussion}

This study replicated the initial validation study on the German PABS-PT to reexamine the factor structure of the questionnaire (Laekeman et al., 2008). EFA and CFA were conducted on different item pools to examine the underlying structure of the scale and to test the fit of the hypothesized 2-factor model of the German PABSPT. Our findings from EFA and CFA do not support the validity of the 2-factor model that was previously considered as an adequate representation of the German PABSPT. The results of our study are inconsistent with those of the previous validation on the German PABS-PT (Laekeman et al., 2008) and also differ from other factor analyses on the measurement scale (Dalkilinc et al., 2015; Eland et al., 2017; Houben et al., 2005; Mutsaers et al., 2014; Ostelo et al., 2003). Thus, the results may indicate a need for caution in using the PABS-PT to measure physiotherapists' orientation toward LBP, particularly for testing the magnitude of their biopsychosocial orientation.

The aim of our study was to replicate the previous validation study on the German PABS-PT. Therefore, we recruited study participants similar to those in the initial study (Laekeman et al., 2008). Both samples consisted of general physiotherapists, mainly specializing in manual therapy, along with undergraduate physiotherapy students. Although the number of participants included in the analysis was larger in our study ( $N=398$ vs 274 ), the overall response rate was lower (37 vs $79 \%$ ) compared with the study of Laekeman et al. (2008)(Laekeman et al., 2008). Nevertheless, we consider the characteristics of participants in these two validation studies to be comparable.

Inconsistencies in the performance of the measurement scale were found between the present and the previous validation of the German language version (Laekeman et al., 2008). Laekeman et al. (2008) excluded 14 measured items from EFA because of a skewness exceeding \pm 1.0 (Laekeman et al., 2008). We found only eight items that met this criterion (see Table 3). Indeed, inconsistencies in the performance of the PABS-PT items could be expected because of differences in the number of items excluded from EFA across other studies (see Table 1). The performance of the scale indicates that the German 36-item PABS-PT includes several items that should be 
revised or discarded from the scale. Furthermore, the results of EFA on the 36-item scale estimated the factor structure of the measurement scale that was used in the present, and in previous, factor analyses of the PABS-PT (Dalkilinc et al., 2015; Eland et al., 2017; Houben et al., 2005; Laekeman et al., 2008; Mutsaers et al., 2014). Overall, results from the German 36 -item PABS-PT indicates that a 2-factor model may not describe the underlying structure of this scale and that the 36 -item scale should be revised.

Previous validation studies of the PABS-PT selected different items for factor analyses to uncover the underlying relationships between latent common factors and measured items (Dalkilinc et al., 2015; Eland et al., 2017; Houben et al., 2005; Laekeman et al., 2008; Mutsaers et al., 2014). The previous validation study on the German PABS-PT included 17 items for EFA and identified two common factors, but these two factors influenced only 14 PABS-PT items. In our study, we replicated EFA on the same 17-item pool, but no method for determining the number of factors to retain suggested retaining only two factors for the final model. The scree plot of the 17 eigenvalues indicated one large factor that might be consistent with the factor 1 "biomedical" factor in other studies (Dalkilinc et al., 2015; Eland et al., 2017; Houben et al., 2005; Laekeman et al., 2008; Mutsaers et al., 2014; Ostelo et al., 2003). This factor seemed to be quite robust regarding the number of significant loadings to the measured items. However, our results do not indicate the validity of a 2 -factor model of the German PABS-PT. The negative results of the CFA further support the proposal that a 2-factor model does not fit the selected PABS-PT items. Therefore, while the PABS-PT may include a biomedical factor that relates to items of the scale, our findings do not support the assumption that the PABS-PT includes one additional biopsychosocial factor.

Previous EFA studies of the PABS-PT applied problematic methods for determining the number of factors to retain. These studies used several different methods; however, the final decision of the researchers to specify a 2-factor model was based on the graphic scree test (Houben et al., 2005; Laekeman et al., 2008; Mutsaers et al., 2014). For example, in the EFA study on the Norwegian PABS-PT, parallel analysis suggested the extraction of four factors (Eland et al., 2017). The authors, however, retained only two factors based on the examination of the scree plot. Parallel analysis is considered as an objective and adequate method in EFA 
(Leandre R. Fabrigar et al., 1999; Henson \& Roberts, 2006; Ruscio \& Roche, 2012). In contrast, the graphic scree test has been criticized because of its subjectivity (Leandre R. Fabrigar et al., 1999; Zwick \& Velicer, 1986). Errors in the selection of the number of factors in a model can have substantial effects on the results obtained from EFA (Cattell, 1978). EFA provides several different methods for determining the number of factors that should be included in the model. However, the factor analysis approach is primarily data-driven (L. R. Fabrigar \& Wegener, 2012). Therefore, decisions on the number of factors to retain should be based on objective methods such as parallel analysis.

Limitations of the present study include the low response rate, which might be explained by the strategy used to distribute the questionnaire, whereby contact details were derived from an online database without any personal contact between the researchers and the participants. The low response rate limits the conclusions that can be drawn from this validation study, since it might have led to a sampling bias because physiotherapists with a strong biomedical treatment orientation might not have responded to the invitation to take part in the survey (Laekeman et al., 2008). Another limitation might be the inclusion of students in this study. Students have very limited experience in managing patients with LBP. This could have influenced the performance of the PABS-PT in our study. However, in doing this we accurately replicated the previous validation study of the German PABS-PT which included undergraduate physiotherapy students. Additionally, this study did not include a comparison with other related measurements. Testing the correlation between the PABS-PT and another valid measurement of a theoretically similar construct would have allowed conclusions regarding the convergent validity of the PABS-PT. Further tests on the construct validity and criterion validity of the scale would benefit further development of the questionnaire.

The results of this study indicate that caution should be applied when using the PABS-PT to test the treatment orientation of healthcare providers toward LBP, particularly the magnitude of a biopsychosocial orientation. Some items of the PABSPT might be adequate to measure aspects of a biomedical orientation. These biomedical items could be used in future research to establish cut-off scores for identifying individuals with a strong biomedical orientation. The multifactorial structure of the PABS-PT may also indicate that a biopsychosocial orientation of healthcare 
provider cannot be captured by a single common factor. The dimensionality, or components, of a biopsychosocial orientation toward LBP would be interesting to explore further in future research. Additionally, it would be relevant to investigate how behavioral and affective dimensions relate to physiotherapists' attitudes and beliefs. According to the tripartite model of attitudes, attitudes have distinct cognitive, affective and behavioral components (Crites, Fabrigar, \& Petty, 1994; Wilson, Lindsey, \& Schooler, 2000). It is likely that the content validity of the PABS-PT can be improved by developing more items that capture the affective and behavioral components of attitudes. Furthermore, it might be interesting to explore how or whether components of a biopsychosocial orientation can be observed in the behavior of physiotherapists in clinical practice. Having a suitably robust measure of the PABS-PT and similar scales is necessary given the potential of such scales to assess the beliefs and attitudes of clinicians toward influencing their clinical behavior and patient outcomes. Further work is therefore needed to revise and enhance the PABS-PT.

\section{Acknowledgements}

The authors thank all the physiotherapists and students who participated in this study, and Maria-Anna Laekeman for providing the German PABS-PT version and additional study documents.

\section{Declaration of Interest}

The authors declare no conflict of interest. 


\subsection{References}

- Insert references here - 